\title{
Using functional genomics to decipher the complexity of microbial pathogenicity
}

\author{
Maisem Laabei ${ }^{1} \cdot$ Ruth $_{\text {Massey }}{ }^{1}$ (i)
}

Received: 11 December 2015 / Revised: 2 February 2016 / Accepted: 3 February 2016 / Published online: 22 February 2016

(C) The Author(s) 2016. This article is published with open access at Springerlink.com

\begin{abstract}
From the first identification of bacteria as a causative agent of disease, researchers have been developing methods and techniques to understand their pathogenic processes. For decades, this work has been limited to looking at a small number of genetically manipulatable isolates in in vitro assays or animal models of infection. Despite these limitations such work has facilitated the development of successful therapeutic strategies, most notably vaccines that target specific virulence-related features. There are however many antimicrobial resistant pathogens for which vaccination strategies have not worked, as we simply do not know enough about how they cause disease. We are however at the dawn of a new era in the study of microbial pathogenicity, where large collections of bacteria isolated directly from human infections can be sequenced and assayed to identify the bacterial features that affect disease severity in humans. Here, we describe our attempt to perform such a study focussed on the major human pathogen Staphylococcus aureus, which demonstrates the step changes such approaches can make to understanding microbial pathogenicity.
\end{abstract}

Keywords Staphylococcus aureus · Toxicity · GWAS · Evolutionary trade-offs · Bacteraemia

Communicated by M. Kupiec.

Ruth Massey

r.c.massey@bath.ac.uk

1 Department of Biology and Biochemistry, University of Bath, Bath BA2 7AY, UK
Staphylococcus aureus is the primary cause of a wide range of human infections, including septicaemia, abscesses and endocarditis (Lowy 1998). Although the rates of methicillin-resistant $S$. aureus (MRSA) are declining in certain European hospitals, a recent study showed that the incidence of MRSA bacteraemia was greater than $25 \%$ in over one-third of European countries (Johnson 2011). In the United States, the rate of MRSA infection has decreased since 2006 (Klein et al. 2007); however, the proportion of those infections caused by the highly virulent, sequence type 8, USA300 clone has risen sharply (Jarvis et al. 2012; Rhee et al. 2015). USA300 is the predominant clone causing community-acquired infections in otherwise healthy individuals, and is now considered endemic in many US communities and hospitals (David and Daum 2010; Rhee et al. 2015). Even with increased surveillance, improved patient management and treatment regimes, $S$. aureus remains a major public health concern (Johnson 2011; Jarvis et al. 2012). As a response to the severe threat $S$. aureus possesses to global human health, a deeper understanding of its pathogenesis at the genetic and molecular level is warranted.

Staphylococcus aureus is both a major human pathogen and resident of the normal microflora. For decades, it has been considered an opportunistic pathogen, where breaches in host immunity are considered the route through which it transitions from being a coloniser to an invasive pathogen. This suggests that disease onset and severity are wholly driven by the host, where given identical opportunity two genetically distinct $S$. aureus isolates will cause identical disease. However, the fact that we have globally successful clones of $S$. aureus circulating and hyper-virulent strains causing a significant burden of disease suggests that specific bacterial features contribute significantly to the onset and severity of disease. Until we understand the effect of 
these on disease in humans we will not have a full picture of this microbe's pathogenicity.

Staphylococcus aureus possesses an extensive repertoire of virulence factors facilitating its dual life-style as a commensal and a pathogen (Foster and Hook 1998; Vandenesch et al. 2012; Vorkapic et al. 2015), where the secretion of toxins is classically considered an essential element in the development of infection (Dinges et al. 2000). The staphylococcal scientific literature is abundant with many excellent studies involving the mechanism of action, host receptor tropism and regulation of the many toxins secreted by $S$. aureus (Vandenesch et al. 2012; Ibarra et al. 2013; DuMont and Torres 2014). At present, we are at a stage where it is affordable to rapidly and accurately sequence large numbers of clinical isolates, resulting in new opportunities to study pathogen evolution, epidemiology and antibiotic resistance (Didelot et al. 2012). The question that has been presented is whether this revolution in next-generation sequencing and abundance in genomic information can aid in our understanding of the fundamental basis of $S$. aureus virulence.

As the secretion of toxins is an integral part of the $S$. aureus virulence repertoire, we focussed on investigating the molecular and genetic basis of toxin secretion. Here, we combined whole-genome sequencing and phenotypic data, developing a genome-wide association study (GWAS) to detail how polymorphisms affect bacterial cytotoxicity. In two recent publications, we document the functional genomics approach we undertook to determine which mutations affected this phenotype. In our first paper 'Predicting the virulence of MRSA from its genome sequence' (Laabei et al. 2014), we explored the use of GWAS to identify genes, acting singularly or in epistasis, which we predicted to have an impact of toxicity, using the globally important, widely-disseminated ST239 lineage (Laabei et al. 2014). From our list of 121 statistically significant polymorphisms affecting toxicity, we sought to functional verify 13 of these loci, resulting in the description of four previously uncharacterised regions of the ST239 genome, that when disrupted significantly impact on toxicity. Additionally, by adopting a machine learning approach using a subset of these polymorphisms (unique SNPs and one SNP from each group of SNPs in linkage disequilibrium) we were able to make predictions on the toxicity of these strains using sequencing data with high accuracy $(>85 \%)$ (Laabei et al. 2014).

Staphylococcus aureus gene expression is tightly controlled and has evolved to be energetically efficient. The secretion of toxins, generally considered to be positively associated with increased disease severity, comes at a high energetic price and is readily switched off in vitro (Somerville et al. 2002) with recent observational studies suggesting that this also occurs in vivo (Fowler et al. 2004;
Rose et al. 2015). In our most recent paper, 'Evolutionary trade-offs underlie the multifaceted virulence of $S$. aureus' (Laabei et al. 2015), we adopted our functional genomics approach to understand and define mutations associated with this reduction in toxicity. Therein, we describe the elucidation of six novel loci that when mutated significantly decrease toxicity, highlighting the complex nature of $S$. aureus toxin regulation (Laabei et al. 2015). The decrease in toxicity was significantly associated with strains originating from bacteraemia, compared to strains from skin and soft tissue infections (SSTIs) or carriage strains. Previous groups showed that clinical strains isolated from the blood had increased defective mutations in the accessory gene regulator (Agr) operon, the main quorum sensing system which regulates toxins in $S$. aureus and had increased resistance to vancomycin (Sakoulas et al. 2005). Interestingly, in this collection of isolates there was no difference in the number of Agr mutations present in the carriage/ SSTI group compared to the bacteraemia group, and no significant difference between vancomycin resistance between the two groups (data not published).

We further investigated several hypotheses as to why a decrease in toxicity may be selected for and associated with bacteraemia. By measuring growth dynamics and relative fitness, we found that low-toxic isolates were significantly more fit when grown in the presence of serum than hightoxic isolates. Given the extreme bottleneck bacteria have to traverse to establish a bloodstream infection, our hypothesis is that a reduction in toxicity by mutation of various genes increases its relative fitness compared to high-toxic strains due to the energy cost of toxin production.

The discovery and elucidation of novel virulence regulators has been an immensely important feature of understanding $S$. aureus pathogenesis. To the best of our knowledge there are approximately 30 known virulence regulators, including two-component systems, DNA-binding proteins and regulatory RNAs (Priest et al. 2012; Tuchscherr and Loffler 2015). Virulence factors' expression in $S$. aureus occurs in a temporal growth-phase-dependent manner employing multiple arms of this complex regulon at any one time. In the past 2 years, as a result of our functional genomics approach a further 10 genetic loci have been shown to be affected by $S$. aureus virulence, specifically toxicity. From our unpublished work, we have identified a further three loci important in the regulation of toxicity, of which we are currently characterising. Following with this theme, and focusing on $S$. aureus biofilm activity, we have identified two novel regions that increase biofilm formation following gene inactivation. These preliminary forays into applying functional genomics approaches to microbial pathogens clearly illustrate how fruitful this can be.

The functional genomics approach we have developed makes use of assaying large numbers of clonally related 
clinical strains. This methodology has not only allowed us to make significant leaps in understanding the vast regulon that affects toxicity, and virulence in general, but has highlighted that intra-clonally, strains have highly variable toxicity levels, a feature that has not been shown to this extent before. The functional genomics approach allows us to better understand pathogen biology, shown here by the discovery of the trade-off which exists between $S$. aureus toxicity and fitness in serum which we hypothesise is an essential step in the progression of bacteraemia. As we are entering the next-generation sequencing era, whereby the cost of sequencing a single strain has reduced dramatically, whilst the accuracy has increased significantly, we desperately need a strategy to understand this abundance in genomic information and how it impacts on pathogen behaviour. By combining large-scale sequencing with comprehensive phenotypic studies, we can begin to map the entire virulence pathway, identifying virulence signatures responsible for hyper-virulence. These strategies may play a central role in developing the holy-grail of patient care, personalised medicine, whereby detailed patient data and bacterial genome sequences will be used in combination to optimise treatment and improve the outcome for patients.

Open Access This article is distributed under the terms of the Creative Commons Attribution 4.0 International License (http://creativecommons.org/licenses/by/4.0/), which permits unrestricted use, distribution, and reproduction in any medium, provided you give appropriate credit to the original author(s) and the source, provide a link to the Creative Commons license, and indicate if changes were made.

\section{References}

David MZ, Daum RS (2010) Community-associated methicillinresistant Staphylococcus aureus: epidemiology and clinical consequences of an emerging epidemic. Clin Microbiol Rev 23:616-687

Didelot X, Bowden R, Wilson DJ, Peto TE, Crook DW (2012) Transforming clinical microbiology with bacterial genome sequencing. Nat Rev Genet 13:601-612

Dinges MM, Orwin PM, Schlievert PM (2000) Exotoxins of Staphylococcus aureus. Clin Microbiol Rev 13:16-34 (table of contents)

DuMont AL, Torres VJ (2014) Cell targeting by the Staphylococcus aureus pore-forming toxins: it's not just about lipids. Trends Microbiol 22:21-27

Foster TJ, Hook M (1998) Surface protein adhesins of Staphylococcus aureus. Trends Microbiol 6:484-488

Fowler VG Jr, Sakoulas G, McIntyre LM, Meka VG, Arbeit RD, Cabell CH, Stryjewski ME, Eliopoulos GM, Reller LB, Corey GR, Jones T, Lucindo N, Yeaman MR, Bayer AS (2004) Persistent bacteremia due to methicillin-resistant Staphylococcus aureus infection is associated with agr dysfunction and low-level in vitro resistance to thrombin-induced platelet microbicidal protein. J Infect Dis 190:1140-1149
Ibarra JA, Perez-Rueda E, Carroll RK, Shaw LN (2013) Global analysis of transcriptional regulators in Staphylococcus aureus. BMC Genom 14:126

Jarvis WR, Jarvis AA, Chinn RY (2012) National prevalence of methicillin-resistant Staphylococcus aureus in inpatients at United States health care facilities, 2010. Am J Infect Control 40:194-200

Johnson AP (2011) Methicillin-resistant Staphylococcus aureus: the European landscape. J Antimicrob Chemother 66(Suppl 4):iv43-iv48

Klein E, Smith DL, Laxminarayan R (2007) Hospitalizations and deaths caused by methicillin-resistant Staphylococcus aureus, United States, 1999-2005. Emerg Infect Dis 13:1840-1846

Laabei M, Recker M, Rudkin JK, Aldeljawi M, Gulay Z, Sloan TJ, Williams P, Endres JL, Bayles KW, Fey PD, Yajjala VK, Widhelm T, Hawkins E, Lewis K, Parfett S, Scowen L, Peacock SJ, Holden M, Wilson D, Read TD, van den Elsen J, Priest NK, Feil EJ, Hurst LD, Josefsson E, Massey RC (2014) Predicting the virulence of MRSA from its genome sequence. Genome Res 24:839-849

Laabei M, Uhlemann AC, Lowy FD, Austin ED, Yokoyama M, Ouadi K, Feil E, Thorpe HA, Williams B, Perkins M, Peacock SJ, Clarke SR, Dordel J, Holden M, Votintseva AA, Bowden R, Crook DW, Young BC, Wilson DJ, Recker M, Massey RC (2015) Evolutionary trade-offs underlie the multi-faceted virulence of Staphylococcus aureus. PLoS Biol 13:e1002229

Lowy FD (1998) Staphylococcus aureus infections. N Engl J Med 339:520-532

Priest NK, Rudkin JK, Feil EJ, van den Elsen JM, Cheung A, Peacock SJ, Laabei M, Lucks DA, Recker M, Massey RC (2012) From genotype to phenotype: can systems biology be used to predict Staphylococcus aureus virulence? Nat Rev Microbiol 10:791-797

Rhee Y, Aroutcheva A, Hota B, Weinstein RA, Popovich KJ (2015) Evolving Epidemiology of Staphylococcus aureus Bacteremia. Infect Control Hosp Epidemiol 36:1417-1422

Rose HR, Holzman RS, Altman DR, Smyth DS, Wasserman GA, Kafer JM, Wible M, Mendes RE, Torres VJ, Shopsin B (2015) Cytotoxic virulence predicts mortality in nosocomial pneumonia due to methicillin-resistant Staphylococcus aureus. J Infect Dis 211:1862-1874

Sakoulas G, Eliopoulos GM, Fowler VG Jr, Moellering RC Jr, Novick RP, Lucindo N, Yeaman MR, Bayer AS (2005) Reduced susceptibility of Staphylococcus aureus to vancomycin and platelet microbicidal protein correlates with defective autolysis and loss of accessory gene regulator (agr) function. Antimicrob Agents Chemother 49:2687-2692

Somerville GA, Beres SB, Fitzgerald JR, DeLeo FR, Cole RL, Hoff JS, Musser JM (2002) In vitro serial passage of Staphylococcus aureus: changes in physiology, virulence factor production, and agr nucleotide sequence. J Bacteriol 184:1430-1437

Tuchscherr L, Loffler B (2016) Staphylococcus aureus dynamically adapts global regulators and virulence factor expression in the course from acute to chronic infection. Curr Genet 62:15-17

Vandenesch F, Lina G, Henry T (2012) Staphylococcus aureus hemolysins, bi-component leukocidins, and cytolytic peptides: a redundant arsenal of membrane-damaging virulence factors? Front Cell Infect Microbiol 2:12

Vorkapic D, Pressler K, Schild S (2016) Multifaceted roles of extracellular DNA in bacterial physiology. Curr Genet 62:71-79 